\title{
Freezing line of the Lennard-Jones fluid: a Phase Switch Monte Carlo study
}

\author{
Graham C. McNeil-Watson and Nigel B. Wilding \\ Department of Physics, University of Bath, Bath BA2 7AY, United Kingdom
}

(Dated: December 3, 2018)

\begin{abstract}
We report a Phase Switch Monte Carlo (PSMC) method study of the freezing line of the LennardJones (LJ) fluid. Our work generalizes to soft potentials the original application of the method to hard sphere freezing, and builds on a previous PSMC study of the LJ system by Errington (J. Chem. Phys. 120, $3130(2004))$. The latter work is extended by tracing a large section of the LennardJones freezing curve, the results for which we compare to a previous Gibbs-Duhem integration study. Additionally we provide new background regarding the statistical mechanical basis of the PSMC method and extensive implementation details.

PACS numbers: 64.70Fx, 68.35.Rh
\end{abstract}

\section{INTRODUCTION AND BACKGROUND}

The freezing of a disordered fluid into an ordered crystalline solid undoubtedly represents one of the most spectacular examples of thermodynamics in action. But despite its ubiquity and familiarity, key aspects of the phenomenon remain poorly understood across a variety of systems [1]. Principal among the reasons for this is the difficulty of obtaining accurate simulation estimates for fluid-solid phase coexistence properties. In this section we outline the most commonly used contemporary approaches for tracing freezing boundaries, identify their key strengths and weaknesses, and highlight recent developments in the search for improvements.

The staple method for locating fluid-solid coexistence is thermodynamic integration (TI) [1-3]. Here, the free energy of each phase (fluid (F) and crystalline solid (CS)) is computed for states covering a range of densities, using integration methods which connect their thermodynamic properties with those of reference states of known free energy. The two branches of the free-energy are then matched to determine the freezing parameters. While there is much to commend TI (principally its simplicity) it turns out to be less than ideal in a number of respects [4]. These have been documented in detail elsewhere (see eg. [5]); but briefly, the main difficulty is one of identifying a suitable reference state together with a reversible phase space pathway by which it can be reached; a poor choice of integration path may encounter singularities -both real and artificial [6]- which compromise the calculation. Additionally, corrections may be required to allow for the fact that the path does not quite reach the idealized reference state [2, 7]. Moreover, the method focuses on the absolute free energies of the phases [8], rather than the quantity of interest -their free energy difference. Finally, there currently exists no reliable and comprehensive method for estimating errors on phase boundaries computed via TI.

Obtaining a whole F-CS phase boundary using TI is a computationally challenging task. However, knowledge of one point on the F-CS coexistence boundary (obtained, for instance, by using TI) can be used to bootstrap a Gibbs-Duhem integration (GDI) scheme to trace the entire curve without further calculation of free energies. The GDI method [9-11] exploits the generalized Clausius-Clapeyron equation to express the slope of the phase boundary entirely in terms of single-phase averages. This is clearly a virtue since it avoids the need for two-phase sampling. However, without any 'reconnection' of the two configuration-spaces at subsequent simulation state points, the GDI approach offers no feedback on integration errors. Since there will generally exist a band of metastable states on each side of the phase boundary, it is possible for the integration to wander significantly from the true boundary with no indication that anything is wrong. As a result, the calculation of meaningful uncertainties is problematic. GDI has been used in a number of studies, most notably in the context of freezing of hard and soft spheres [12].

More recently, attention has shifted to developing methods for tackling the problem of locating F-CS coexistence via direct measurements of free energy differences. To do so, one must construct a reversible sampling path between the phases. The obvious "physical" path, traversing the region where both phases coexist -whilst practicable when the two phases share the same symmetry (see eg. ref. [13]) -turns out to be computationally problematic in the F-CS context. The main reasons for this are the large degree of metastability of the two phases, the extended timescale for the crystallization process, and the tendency of any crystal formed to exhibit vacancies, interstitials and other defects. Accordingly, recent effort has focussed on trying to identify alternative inter-phase routes for the F-CS problem.

One such alternative route, called constrained fluid- $\lambda$ integration has been proposed by Grochla[14] and extended by Elke et al [15]. It involves the controlled transformation of the fluid phase to the solid phase in a series of stages. During the initial stage the fluid is gradually changed to a weakly interacting fluid by reducing the strength of interparticle interactions. At the next stage, a set of Gaussian potential wells of prescribed width are switched on at the sites of a crystalline lattice of the appropriate symmetry. Simultaneously, the volume of the system is gradually changed from a value typical of the fluid to one typical of the solid. The particles of the weakly interacting fluid are 
then allowed to diffuse to the lattice sites, where they are captured by the Gaussian wells. The final stage involves gradually restoring the particle interactions to full strength, whilst simultaneously switching off the Gaussian potential wells. Integration of free energy derivatives is used to estimate the free energy difference along the path.

The constrained fluid- $\lambda$ integration method was tested by application to the Lennard-Jones fluid where it was used to determine two coexistence state points. This test led the author to conclude that the method "cannot be said to be computationally efficient" [14], at least in its present form. This finding presumably reflects the rather long and fragmented nature of the inter-phase path. Although each of the stages of the path was initially reported to be reversible [15, 16], further application of the method [16] found this to be contingent on the correct tuning (via trial and error) of four separate constants relating to the characteristics of the Gaussian potential wells. Indeed, it is not clear to us that one can generally expect a-priori that the stated path will be trouble-free; for example, it seems hard to rule out that the transformation of the original fluid to a weakly interacting fluid might intersect a first order (pseudo) liquid-vapor transition. Furthermore we speculate that for large systems the reliance on particle diffusion to translate particles to unoccupied lattice sites may become problematic.

Very recently, Mastny and de Pablo [17] have proposed a method based on measurements of the density of states, which aims to directly estimate the free energy difference of F and CS phases. The rationale for the method is its authors' assertion that: "To connect the free energy of the solid and liquid phases, there must exist a portion of energy and volume space that can be simultaneously sampled by both solid and liquid phases". To exploit this premise, a series of simulations of the Lennard-Jones system were performed, each restricted to -and sampling the configuration space of- one of a set of overlapping "windows" in the space of the total energy $E$ and volume $V$. Successive windows were positioned appropriately such as to form a path linking the two well-separated regions of $E, V$ associated with the typical configurations of the respective phases. Along this path, a central range of $E, V$ was indeed reported to be found for which both liquid and solid phase configurations could be sampled. A simple average [18] of the solid phase and liquid phase density of states was accumulated in the central range and joined for continuity to the measured forms of the liquid and solid density of states on either side.

In our opinion, the existence of a range of $E$ and $V$ that can be sampled by both liquid and solid phases is insufficient to connect the respective branches of the density of states because these are (like their underlying sets of characteristic configurations), fundamentally distinct. Instead, for a proper connection, the phases must be linked via a continuous (and reversible) path through configuration space. In the context of the method of Mastny and de Pablo, this necessitates repeated (back and forth) transitions between the two pure phases. Since no mention is made in ref. [17] of any such transitions, the validity of the method would appear to be questionable [19].

Another very recent approach to the F-CS problem, which bears some resemblance to that of ref. [17], is the "multiNpH" method of Escobedo [20]. Here a path is constructed in the enthalpy of the system, spanning the range of values between those typical of the respective equilibrium phases at some prescribed pressure. The system temperature is ascertained at each point along the path, and exhibits a discontinuity at some value of the enthalpy as the favored phase of the system changes. TI with respect to the temperature variations along the path yields the free energy difference. The problem reported with this method was that it was apparently necessary for equilibration purposes to initialize the system in the CS phase at all points along the enthalpy path. However, it was difficult to be sure whether, for a given enthalpy value, the system had relaxed to the phase of minimum free energy, and hence whether the TI result was unbiased. This seems to us to be a manifestation of the familiar drawback of TI, namely the difficulty of finding a reversible integration path.

Phase Switch Monte Carlo (PSMC) [21] is a further relatively recent method designed to directly link F and CS phases via a reversible sampling path. The method builds on previous work [22] in which it was demonstrated that the configuration spaces associated with two phases of a many-body system can both be visited in a single Monte Carlo simulation, by harnessing extended sampling methods to facilitate a direct switch from one phase to the other. The method, which is quite generally applicable, was initially deployed in a study of hard sphere freezing [21]. Subsequently, it was applied to soft potentials by Errington [23], who used it to calculate two points on the freezing line of the Lennard-Jones system for a number of system sizes.

The present paper complements and extends Errington's PSMC study of the LJ system. It is organized as follows. In sec. II we review some of the key results in the statistical mechanical formulation of F and CS phase configurational weights, and show how to construct, on this basis, a theoretical framework for computational estimation of free energy differences. Sec III describes how this framework is exploited in principle, and realized in practice, by the PSMC method. An application of the method to the Lennard-Jones system is reported in sec. IV where we present estimates of large portions of the freezing curve for a number of system sizes. These we compare with the results of a previous GDI study, bootstrapped using TI at a single coexistence point. Finally sec. V details our conclusions and the prospects for further applications. 


\section{STATISTICAL MECHANICS}

Within the framework of statistical mechanics, the free energy of a given phase is expressible in terms of its configurational weight. Information regarding this weight can be accumulated via a simulation in the course of an exploration (sampling) of the microstates of the phase. While for fluid phases such an exploration encounters no great obstacles, a complication arises in the case of crystalline solids. Specifically, it transpires that the relevant phase space of the solid is effectively fragmented into a number of mutually inaccessible regions. Each fragment corresponds to a distinct permutation of the particles with respect to the lattice sites. The solid phase configurational weight can only be estimated for the single fragment in which it is initiated. Since on symmetry grounds the weight contribution of every fragment is identical, the overall configurational weight of the solid is obtained by multiplying the measured weight of one fragment by the number of fragments. In this section we first show how to correctly count this fragment number. We then proceed to describe how the ratio of configurational weights of two phases is related to the ratio of their a priori probabilities (a quantity directly accessible to simulation) and thence to the free energy difference.

Consider a periodic system of $N$ classical particles confined to a volume $V$, variable under a prescribed constant external pressure $p$, and in equilibrium with a heat bath at prescribed constant temperature $T$. Within this constant$N p T$ ensemble the configurational weight of a phase may be written as

$$
\mathcal{Z}_{\gamma}(N, p, T)=\int_{0}^{\infty} d V e^{-p V} Z_{\gamma}(N, V, T)
$$

Here, $\gamma$ labels the phase, while

$$
Z_{\gamma}(N, V, T)=\prod_{i=1}^{N} \int_{V, \gamma} d \mathbf{r}_{i} e^{-\Phi(\{\mathbf{r}\})},
$$

with $\Phi$ the configurational energy [24]. The $\gamma$-label on the integral stands for some configurational constraint that picks out configurations $\{\mathbf{r}\}$ that 'belong' to phase $\gamma$. In the present work we shall be concerned with phases which are either fluid $(\gamma=\mathrm{F})$ or crystalline solid $(\gamma=\mathrm{CS})$ in character, and choose to formulate the constraint in a form which reflects the situation actually encountered in Monte Carlo simulations of single phases. Specifically, let $\mathbf{R}_{1}^{\gamma} \ldots \mathbf{R}_{N}^{\gamma} \equiv\{\mathbf{R}\}^{\gamma}$ denote some representative configuration of phase $\gamma$. Then the constraint may be regarded as picking out those configurations which can be reached from $\{\mathbf{R}\}^{\gamma}$ on the simulation timescale. The timescale is presumed to be sufficiently long to explore 'one phase' but still short compared to (unaided) inter-phase traverses.

It is convenient to use the sites defined by $\{\mathbf{R}\}^{\gamma}$ as the origins of the particle coordinates. Thus we define a set of displacement vectors $\{\mathbf{u}\}$ where

$$
\mathbf{u}_{i} \equiv \mathbf{r}_{i}-\mathbf{R}_{i}^{\gamma},
$$

and write $\Phi \gamma(\{\mathbf{u}\}) \equiv \Phi\left(\left\{\mathbf{R}^{\gamma}+\mathbf{u}\right\}\right)$. In the case of the fluid phase, particles have the run of the entire system and hence all contributing configurations are reachable from any one; we may write simply

$$
Z_{F}(N, V, T)=\prod_{i=1}^{N} \int_{V,\{\mathbf{R}\}_{F}} d \mathbf{u}_{i} e^{-\Phi_{F}(\{\mathbf{u}\})},
$$

where $\{\mathbf{R}\}^{F}$ is some specific but arbitrary fluid configuration, which can be selected at random in the course of MC exploration of the fluid phase.

For the crystalline phase it is natural to choose $\{\mathbf{R}\}^{C S}$ to define the sites of a lattice of the appropriate symmetry and scale. But here one must recognize that the complete CS configuration space actually comprises a number of distinct mutually inaccessible fragments [25] corresponding essentially to the different permutations of particles amongst lattice sites. By symmetry each fragment should contribute equally to the configurational weight. But in general, the typical timescale for particle interchanges between lattice sites greatly exceeds the accessible simulation timescale. In these circumstances MC simulation will visit (and thus count) only the states within the fragment in which it is initiated. The total configurational weight of the CS phase must therefore be obtained by multiplying the measured contribution of one fragment by the total number of fragments. Since global translation (permitted by the boundary conditions) ensures that one fragment includes all possible locations of any chosen particle, the number of fragments is the number of ways of assigning the other $N-1$ particles to $N-1$ Wigner-Seitz cells of some underlying notional fixed lattice [26]. This number is $(N-1)$ !. Thus 


$$
Z_{C S}(N, V, T)=(N-1) ! \prod_{i=1}^{N} \int_{V,\{\mathbf{R}\}^{C S}} d \mathbf{u}_{i} e^{-\Phi_{C S}(\{\mathbf{u}\})} .
$$

In order to make contact with the simulation methodology to be described below, it is expedient to define the total a priori probability of phase $\gamma$. This is given by the ratio of the configurational weight of phase $\gamma$ to the total weight of the two phases [5]:

$$
P(\gamma \mid N, p, T)=\frac{\mathcal{Z}_{\gamma}(N, p, T)}{\mathcal{Z}_{F}(N, p, T)+\mathcal{Z}_{C S}(N, p, T)}
$$

Combining this last equation with eqs. 1, 4 and 5 , allows the ratio of the configurational weights of the two phases to be expressed in terms of the ratio of their a priori probabilities [22]:

$$
\begin{aligned}
\mathcal{R}_{\mathrm{F}, \mathrm{CS}} & \equiv \frac{\mathcal{Z}_{F}(N, p, T)}{\mathcal{Z}_{C S}(N, p, T)}=\frac{P(F \mid N, p, T)}{P(C S \mid N, p, T)} \\
& =\frac{\int_{0}^{\infty} d V e^{-p V} \prod_{i=1}^{N} \int_{V,\{\mathbf{R}\}^{F}} d \mathbf{u}_{i} e^{-\Phi_{F}(\{\mathbf{u}\})}}{(N-1) ! \int_{0}^{\infty} d V e^{-p V} \prod_{i=1}^{N} \int_{V,\{\mathbf{R}\}^{C S}} d \mathbf{u}_{i} e^{-\Phi_{C S}(\{\mathbf{u}\})}}
\end{aligned}
$$

The link between the configurational weight of a phase and its Gibbs free energy is forged by the definition:

$$
\mathcal{Z}_{\gamma}(N, p, T) \equiv e^{-G_{\gamma}(N, p, T)} .
$$

Inserting this into eq. 7, the Gibbs free-energy-density difference between the phases can be written in the (strategically suggestive) form:

$$
\Delta g \equiv g_{C S}(N, p, T)-g_{F}(N, p, T) \equiv \frac{1}{N} \ln \mathcal{R}_{\mathrm{F}, \mathrm{CS}} .
$$

Eqs. 7 and 9 provide a foundation for MC studies of fluid-solid phase coexistence. Their formal promise is as follows: given an inter-phase sampling scheme that visits both the $\mathrm{F}$ and CS phases, $\mathcal{R}_{\mathrm{F}, \mathrm{CS}}$ (and hence $\Delta g$ ) may be obtained as the ratio of the a priori probabilities of the phases. The latter quantity can be estimated simply as the ratio of the number of visits to each phase recorded in the course of a simulation. In order to exploit this framework, however, one must first design a singularity-free phase space path linking the F and CS phases, and then formulate a sampling strategy to traverse it. Phase Switch Monte Carlo is one realization of such a scheme.

\section{PHASE SWITCH MONTE CARLO}

The relative stability of the $\mathrm{F}$ and CS phases is determined by the ratio of the associated configurational weights $\mathcal{R}_{\mathrm{F}, \mathrm{CS}}$ (Eq. 7). To measure that ratio one needs a MC procedure which visits both solid and fluid regions of configuration space in the course of a single simulation run. The phase switch $\mathrm{MC}$ method is a general approach that facilitates such two-phase sampling. Its principal feature is a phase space 'leap' [22] that directly maps a pure phase configuration of one phase onto a pure phase configuration of the other. The motivation for choosing such an interphase route for the F-CS problem is that it circumvents interfacial (mixed-phase) configurations and their attendant sampling difficulties (see sec. I). In this section we describe how to apply the phase switch method in this context.

The key to implementing the phase switch is the representation (eq. 3) of particle coordinates in term of displacements with respect to a representative configuration $\{\mathbf{R}\}^{\gamma}$. By construction, the system may be transformed between the CS and F phase representative configurations simply by switching the vectors $\left(\mathbf{R}_{i}^{F} \rightleftharpoons \mathbf{R}_{i}^{C S} \forall i\right)$. Thus by continuity, any CS (F) configuration 'sufficiently close' to the representative one will also transform to a F (CS) state under this operation [27]. This phase switch can itself be realized as an MC step, so that the phase label $\gamma$ becomes a stochastic variable. By 'sufficiently close' here, we mean that the energy change associated with the phase switch is such as to afford a reasonable chance of acceptance. Fig. 1 provides an illustration of the mechanism.

We term the set of configurations for which the MC phase switch will be accepted the 'gateway' states. In general, however, these gateway states constitute only a small fraction of the total respective configuration spaces of the phases, 


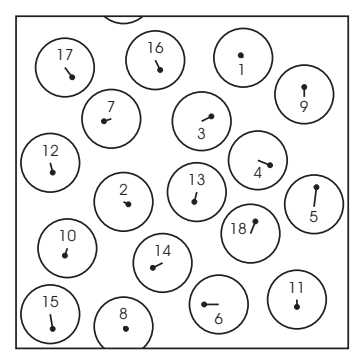

Fluid

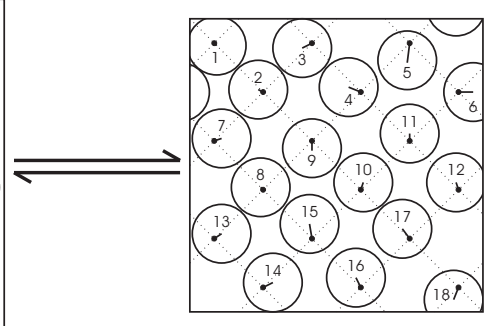

Crystalline solid

FIG. 1: Schematic illustration of the phase switch mechanism. The dots identify the representative sites $\{\mathbf{R}\}^{\gamma}$ in each phase; the displacement vectors $\{\mathbf{u}\}$ connect the centers of the distinguishable (numbered) particles to these sites. The switch operation shown swaps the representative sites of one phase for those of the other phase, whilst maintaining $\{\mathbf{u}\}$ constant. More generally, any desired transformation of $\{\mathbf{u}\}$ (eg. a scaling - see text) can be incorporated in the switch [5]. The particular configuration $\{\mathbf{u}\}$ shown is a "gateway" state (see text) because the magnitude of the effective energy change under the switch is small.

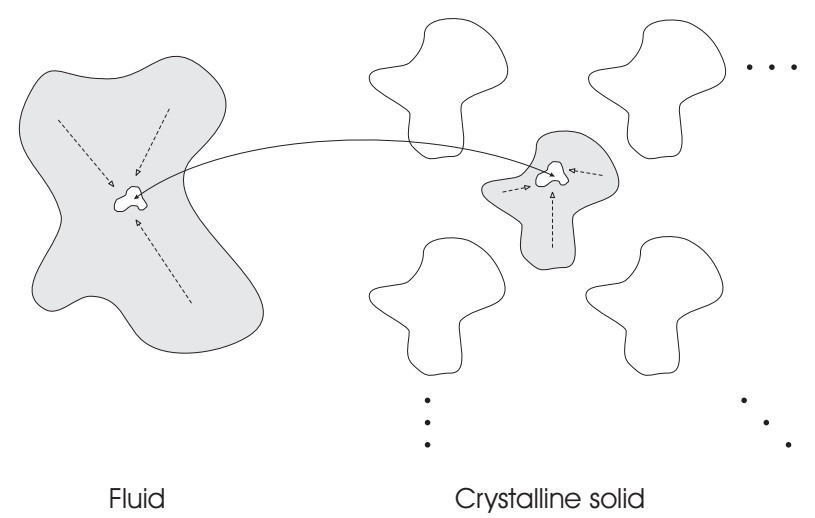

FIG. 2: Schematic illustration of the phase switch operation in terms of the regions of configuration space associated with the F and CS phases. A bias (dashed arrows) is constructed such as to enhance the probability of the subsets of "gateway" states (the white islands) within the single-phase regions, from which the switch operation (the large arrow) will be accepted. Note that the switch accesses only one of the $(N-1)$ ! CS phase space replica fragments (see text).

and consequently will rarely (if ever) be visited on simulation timescales. To ensure effective two-phase sampling, the probabilities with which the gateway states are visited must be enhanced. This can be achieved by appeal to extended (biased) sampling methods. Fig. 2 shows a schematic representation of the procedure.

The bias necessary to promote sampling of the gateway states is administered with respect to an 'order parameter' -some suitably chosen macrovariable of the system. The freedom in selecting this order parameter is circumscribed by the requirement that the associated microstates form a contiguous path through phase space linking the large number of equilibrium (typical) microstates to the relatively small number of gateway states. In the next subsection, we describe one choice of order parameter which has proved itself successful in this regard.

\section{A. Order parameter and extended sampling strategy}

One can devise a variety of order parameters that serve to form a suitable path leading from the equilibrium states to the gateway states. A definition that we have found to be effective is a variant of one originally employed by one of us in ref. [21], and which closely resembles a recent proposal by Errington [23]. Here the order parameter comes in two parts (or modes): 'tether' and 'energy'. The tether mode serves to draw particles close to the representative sites to which they are nominally associated. Then, once all particles are within a prescribed distance of these sites, tether mode switches off and an energy mode order parameter takes over to guide particle to the gateway states for which the phase switch energy cost is sufficiently small to be accepted.

To elaborate, let $M_{m, \gamma}$ denote the order parameter in mode $m$ and phase $\gamma$. Then for tether mode we write $m=\mathcal{T}$ 
and define an associated order parameter

$$
M_{\tau, \gamma}(\{\mathbf{u}\})=\left(\frac{1}{N} \sum_{i=1}^{N} \max \left\{0, \tilde{u}_{i}-\tilde{u}_{c}\right\}\right)^{1 / 2}
$$

where $\tilde{u}_{i}=\left|\mathbf{u}_{i}\right| / V^{1 / 3}$ is the distance of particle $i$ from its lattice site measured in units of the box length, and $\tilde{u}_{c}$ is a prescribed dimensionless threshold radius. Only particles whose displacement $\tilde{u}_{i}$ exceeds this threshold contribute to $M_{\mathcal{T}, \gamma}$.

The tether mode is active iff $\tilde{u}_{i}>\tilde{u}_{c}$ for at least one particle $i$, i.e. when $M_{\mathcal{T}, \gamma}>0$. Otherwise control hands over to the 'energy' mode $m=\mathcal{E}$; its associated order parameter is defined by

$$
M_{\mathcal{E}, \gamma}(\{\mathbf{u}\})=\operatorname{sgn}\left(\Delta \mathcal{E}_{\gamma^{\prime} \gamma}\right) \ln \left(1+\left|\Delta \mathcal{E}_{\gamma^{\prime} \gamma}\right|\right)
$$

where

$$
\Delta \mathcal{E}_{\gamma^{\prime} \gamma}=\left(\mathcal{E}_{\gamma}-\mathcal{E}_{\gamma}^{\mathrm{ref}}\right)-\left(\mathcal{E}_{\gamma^{\prime}}-\mathcal{E}_{\gamma^{\prime}}^{\mathrm{ref}}\right)
$$

measures the change (under the phase switch operation) of the Hamiltonian $\mathcal{E}_{\gamma}(\{\mathbf{u}\}, V)=\Phi_{\gamma}(\{\mathbf{u}\})+p V$ with respect to its value $\mathcal{E}^{\text {ref }}$ in the representative microstate $\{\mathbf{R}\}^{\gamma}$, with the latter scaled to the instantaneous volume of phase $\gamma$ $[23,28]$. The presence of the logarithm in eq. 11 is designed to moderate the scale of the contribution of the energy cost to $M_{\mathcal{E}, \gamma}$, which might otherwise become excessively large for particles with a strongly repulsive core to their interaction potential.

Given these definitions, and recalling eqs. 4 and 5, the entire region of accessible configuration space can be described by the ensemble

$$
\tilde{\mathcal{Z}}(N, p, T,\{\eta\}) \equiv \sum_{\gamma} \int_{0}^{\infty} d V \prod_{i=1}^{N} \int_{\gamma} d \mathbf{u}_{i} e^{-\mathcal{H}_{\gamma}(\{\mathbf{u}\}, V)},
$$

where $\mathcal{H}_{\gamma}$ is the effective Hamiltonian defined by

$$
\begin{aligned}
\mathcal{H}_{\gamma}(\{\mathbf{u}\}, V)= & \Phi\left(\{\mathbf{u}\},\{\mathbf{R}\}^{\gamma}\right)+p V+\eta_{m, \gamma}(M) \\
& -\delta_{\gamma, C S} \ln (N-1) !
\end{aligned}
$$

Here $\{\eta\}$ represents a set of multicanonical weights [5, 29], associated with the macrostates of the macrovariable $M=M_{m, \gamma}$. The set can usefully be split into four 'branches', one for each combination of mode and phase. The role of the weights is to modify (with respect to the Boltzmann sampling) the probability with which the various $M$ values are visited. More specifically, as discussed below, we tailor their form in order to ensure roughly uniform sampling over the relevant range of $M$.

Simulations in the ensemble described by eq. 13 allow one to measure the multicanonical probability distribution

$$
P(M, V, \mathcal{E}, \gamma \mid N, p, T,\{\eta\}) \equiv \tilde{\mathcal{Z}}^{-1} \prod_{i=1}^{N} \int_{\gamma} d \mathbf{u}_{i} e^{-\mathcal{H}_{\gamma}(\{\mathbf{u}\}, V)} \delta(M-M(\{\mathbf{u}\}, V)) \delta\left(\mathcal{E}_{\gamma}-\mathcal{E}_{\gamma}(\{\mathbf{u}\}, V)\right),
$$

the form of which is accumulated in practice via a simple list (see sec. III B 2). The bias introduced by the multicanonical weights can be unfolded to give the equilibrium distribution (to within an unspecified normalization constant):

$$
P(M, V, \mathcal{E}, \gamma \mid N, p, T) \doteq P(M, V, \mathcal{E}, \gamma \mid N, p, T,\{\eta\}) e^{\eta_{m, \gamma}(M)}
$$

The distribution of any single observable can be readily extracted from $P(M, V, \mathcal{E}, \gamma)$ by integration. Similarly the ratio of configurational weights is obtained as 


$$
\begin{aligned}
\mathcal{R}_{\mathrm{F}, \mathrm{CS}} & =\frac{\mathcal{Z}_{F}(N, p, T)}{\mathcal{Z}_{C S}(N, p, T)} \\
& =\frac{\int d M d V d \mathcal{E} P(M, V, \mathcal{E}, F \mid N, p, T)}{\int d M d V d \mathcal{E} P(M, V, \mathcal{E}, C S \mid N, p, T)}
\end{aligned}
$$

from which the Gibbs free-energy-density difference follows directly via eq. 9.

\section{B. Implementation}

\section{MC moves}

The Monte Carlo procedure we have adopted utilizes four types of update, which we describe below. In implementing these updates, it is computationally expedient to hold one particle fixed at its representative site in each phase. This suppresses the global translation mode in the CS phase and eliminates the need for association swaps (see below) in this phase. Choosing to make the assignment $\mathbf{r}_{i=N} \equiv \mathbf{R}_{i=N}^{\gamma}$ (implying that $u_{i=N}=0$ ), we use $\{\mathbf{u}\}_{\star}$ to represent sets $\{\mathbf{u}\}$ of displacement coordinates which satisfy this condition. Consequences for acceptance probabilities are examined in appendix. A.

The MC procedure for one-phase exploration has three types of update: 'Particle translations', 'Association swaps' and 'Volume moves'.

1. 'Particle translations'. This is a standard MC procedure: a site (identified by one of the vectors in $\{\mathbf{R}\}{ }^{C S}$ or $\{\mathbf{R}\}^{F}$ ) is chosen at random and a candidate state is chosen by incrementing the position coordinate of the particle associated with that site by a random vector whose components are drawn from a zero-mean uniform distribution of prescribed width. This operation changes both $\{\mathbf{r}\}$ and $\{\mathbf{u}\}_{\star}$

2. 'Association swaps'. In this operation we choose two distinct sites at random ( $i$ and $j$ say) and identify the corresponding displacement vectors $\mathbf{u}_{i}$ and $\mathbf{u}_{j}$. The candidate state is defined by the replacements

$$
\begin{aligned}
& \mathbf{u}_{i} \rightarrow \mathbf{u}_{i}^{\prime} \equiv \mathbf{u}_{j}+\mathbf{R}_{j}-\mathbf{R}_{i} \\
& \mathbf{u}_{j} \rightarrow \mathbf{u}_{j}^{\prime} \equiv \mathbf{u}_{i}+\mathbf{R}_{i}-\mathbf{R}_{j}
\end{aligned}
$$

This process can be regarded as a change of association: the particle which was associated with site $j$ is now associated with site $i$ (and vice versa). It changes the representation of the configuration (the coordinates $\{\mathbf{u}\}$ ); but it leaves the physical configuration invariant.

We apply it only to the fluid phase where particles can wander far from their representative sites and need to be reined back in order to reach the gateway states. One may implement association updates in the CS phase too: the simulation can then be thought of as exploring different CS-phase fragments; the factor of $(N-1)$ ! in Eq. 5 is no longer needed and it is no longer necessary to clamp one particle.

3. 'Volume moves'. The volume is also updated in the conventional way, by a random walk of prescribed maximum step size, with particle position coordinates $\{\mathbf{r}\}$ and representative sites $\{\mathbf{R}\}^{\gamma}$ rescaled . Note that we maintain the ratio $V_{\gamma^{\prime}} / V_{\gamma}$ constant throughout, so the notional 'conjugate' phase $\gamma^{\prime}$ also undergoes a dilation by the same factor.

In each of the above cases the transition to the candidate state is accepted (see appendix A) with the probability

$$
\begin{aligned}
& p_{a}\left(\{\mathbf{u}\}_{\star}, V \rightarrow\{\mathbf{u}\}_{\star}^{\prime}, V^{\prime} \mid \gamma\right)= \\
& \quad \min \left\{1, \exp \left[-\Delta \mathcal{H}_{\gamma}+N \ln \left(V^{\prime} / V\right)\right]\right\}
\end{aligned}
$$

with

$$
\Delta \mathcal{H}_{\gamma}=\mathcal{H}_{\gamma}\left(\{\mathbf{u}\}_{\star}^{\prime}, V^{\prime}\right)-\mathcal{H}_{\gamma}\left(\{\mathbf{u}\}_{\star}, V\right)
$$


All three types of MC move may involve a change in the order parameter $M$ and hence a change $\Delta \eta$ in the multicanonical weights implicit in $\mathcal{H}$ (cf. eq. 14). Note that particle translations or association swaps that hand over control from one order parameter mode to another, involve a weight change which is calculated from the respective branches of the weight function. For example, for a hand-over from tether mode to energy mode, in which the order parameter changes from $M_{\mathcal{T}}$ to $M_{\mathcal{E}}$ the appropriate weight change is calculated as

$$
\Delta \eta \equiv \eta_{\mathcal{E}, \gamma}\left(M_{\mathcal{E}, \gamma}\right)-\eta_{\mathcal{T}, \gamma}\left(M_{\mathcal{T}, \gamma}\right)
$$

4 'Inter-phase switch'. The final type of MC update is the phase switch, which entails replacing one set of the representative configuration vectors, $\{\mathbf{R}\}^{\gamma}$ say, by the other, $\{\mathbf{R}\}^{\gamma^{\prime}}$. If the equilibrium densities of the two phases were close to one another it would also be possible to keep the volumes constant in the switch. But that is not the case in general. Accordingly -if the procedure is to be efficient- the switch needs to incorporate an appropriate volume scaling of the system [30]. It is natural to fix that scaling so that a 'typical' volume $\hat{V}_{\gamma}$ of phase $\gamma$ is matched to a 'typical' volume $\hat{V}_{\gamma^{\prime}}$ of phase $\gamma^{\prime}$. In practice we take $\hat{V}_{\gamma}$ to be the mean volume of phase $\gamma$. The switch is accepted (see appendix A) with the probability

$$
\begin{aligned}
& p_{a}\left(\gamma,\{\mathbf{u}\}_{\star}, V \rightarrow \gamma^{\prime},\{\mathbf{u}\}_{\star}^{\prime}, V^{\prime}\right)= \\
& \quad \min \left\{1, \exp \left[-\Delta \mathcal{H}_{\gamma^{\prime} \gamma}+(N+1) \ln \left(\hat{V}_{\gamma^{\prime}} / \hat{V}_{\gamma}\right)\right]\right\}
\end{aligned}
$$

where

$$
\Delta \mathcal{H}_{\gamma^{\prime} \gamma}=\mathcal{H}_{\gamma^{\prime}}\left(\{\mathbf{u}\}_{\star}^{\prime}, V^{\prime}\right)-\mathcal{H}_{\gamma}\left(\{\mathbf{u}\}_{\star}, V\right)
$$

The phase switch occurs only from states in which the energy mode order parameter is active; the associated change in multicanonical weights is

$$
\Delta \eta \equiv \eta_{\mathcal{E}, \gamma^{\prime}}\left(M_{\mathcal{E}, \gamma^{\prime}}^{\prime}\right)-\eta_{\mathcal{E}, \gamma}\left(M_{\mathcal{E}, \gamma}\right)
$$

where $M_{\mathcal{E}, \gamma^{\prime}}^{\prime}=-M_{\mathcal{E}, \gamma}$ are the new and old order parameter values respectively. This weight discontinuity is at our disposal; we tune it so as to cancel the typical contribution to the acceptance probability associated with the switch.

When discussing our results (sec. IV) we shall occasionally refer to a "Monte Carlo sweep". This we take to comprise $N$ trial particle translations, $N$ trial association swaps, 1 trial volume move and 1 trial phase switch.

\section{Data accumulation and histogram extrapolation}

In the course of the simulations, observations are stored as lists $[31] V_{j}, M_{j}, \mathcal{E}_{j}, \gamma_{j}(j=1,2, \ldots)$. Estimates for the distribution of any observables were accumulated by appropriate binning and weighting of the members of the list

$$
P(X \mid N, p, T) \propto \sum_{j} e^{\eta\left(M_{j}\right)} \delta\left(X-X_{j}\right)
$$

with $X=V ; M$; etc.

In analyzing the data, extensive use was made of histogram extrapolation (HE). This method greatly enhances computational efficiency by facilitating the scanning of a region of pressure and temperature around each simulation state point, without recourse to further simulation. To achieve this, HE reweights the data obtained at one state point $(p, T)$ to yield estimates appropriate to another not-too-distant state point $\left(p^{\prime}, T^{\prime}\right)$. The reliable range of the extrapolation in $p$ and $T$ is dependent on the statistical quality of the original data. For further information on the HE technique, we refer the interested reader to ref. [33]. Its utility in the context of tracking a freezing line will be illustrated in sec. IV. 


\section{Weight function construction}

Prior to collection of phase switch data, it is necessary to determine a suitable set of multicanonical weights $\{\eta\}$ to enable the system to pass readily between the equilibrium and gateway states. In practice the requisite weights are defined with respect to the macrostates formed by discretising (binning) the order parameter macrovariable $M=M_{m, \gamma}$. For a given mode $m$ and phase $\gamma$, the bin width $\Delta M$ was chosen such that for all pairs of successive bins $M^{(i)}$ and $M^{(i+1)}=M^{(i)}+\Delta M$, the corresponding weights satisfy $\eta\left(M^{(i+1)}\right) / \eta\left(M^{(i)}\right)<2$. Doing so ensures that the weight associated with any given macrostate is representative of the full range of the underlying macrovariable, thereby guaranteeing a reasonable $\mathrm{MC}$ acceptance rate.

Consideration of eqs. 14 and eqs. 15 shows that the form of the weights that confers equal probability on every macrostate $M$ (and hence ensures that all are well sampled) is $\eta(M)=-\ln P(M)$. For adequate sampling one therefore requires a prior estimate of $P(M)$. A variety of techniques exist for providing such an estimate, some of which are described in refs. [5, 34]. The approach we have utilized in this work is the transition matrix Monte Carlo method (TMMC) [23, 34-36].

TMMC extracts information on macrostate probabilities by focusing on the statistics of transitions between these macrostates. The method proceeds by defining a collection matrix, $\mathrm{C}$, in which information regarding the macrostate transitions is accumulated. At every MC step, the proposed transition is recorded in $\mathrm{C}$ (irrespective of whether or not it is actually accepted) according to:

$$
\begin{aligned}
\mathrm{C}\left(M \rightarrow M^{\prime}\right) & \leftarrow \mathrm{C}\left(M \rightarrow M^{\prime}\right)+a \\
\mathrm{C}(M \rightarrow M) & \leftarrow \mathrm{C}(M \rightarrow M)+(1-a),
\end{aligned}
$$

where $a$ is the acceptance probability of the move under the Hamiltonian $\mathcal{E}_{\gamma}(\{\mathbf{u}\}, V)$ (rather than the effective Hamiltonian $\mathcal{H}_{\gamma}$ ).

The macrostate transition probabilities derive from the collection matrix via:

$$
\mathrm{T}\left(M \rightarrow M^{\prime}\right)=\frac{\mathrm{C}\left(M \rightarrow M^{\prime}\right)}{\sum_{k} \mathrm{C}\left(M \rightarrow M_{k}\right)},
$$

where the denominator on the RHS sums over all possible values of the macrostates. Knowledge of these transition probabilities permits, in turn, an estimate of the probabilities $P(M)$. Although a number of approaches exist (see eg. ref. [23]), for extracting $P(M)$ from $\mathrm{T}$, a simple, yet unbiased and stable method, considers exclusively those transitions which occur between adjacent macrostates (i.e. examines only the diagonal and first off-diagonal elements of T). Application of the 'balance' condition for macrostate transitions [34]:

$$
\frac{P\left(M^{\prime}\right)}{P(M)}=\frac{\mathrm{T}\left(M \rightarrow M^{\prime}\right)}{\mathrm{T}\left(M^{\prime} \rightarrow M\right)}
$$

then permits assignment of the weight function via

$$
\eta\left(M^{\prime}\right)-\eta(M)=-\ln \left(\frac{\mathrm{T}\left(M \rightarrow M^{\prime}\right)}{\mathrm{T}\left(M^{\prime} \rightarrow M\right)},\right)
$$

The weight function was updated in this manner at regular intervals (typically every 1000 Monte Carlo sweeps). Each update allowed the simulation to explore an ever wider range of order parameter values [23]. We note, however, that weight function updates should not be performed too frequently during the weight evolution process because, strictly speaking, they violates detailed balance. Empirically, however, at the stated update interval, the solution was found to be highly accurate and the procedure efficient.

For the purposes of constructing the weight function, we found it beneficial to decompose the full range of $M$ into a number of overlapping 'windows'. Within each window a fragment of the overall weight function was determined and joined for continuity to those of neighboring windows. The weight differences across the three mode transitions (tether to energy mode for each phase, as well as the phase switch itself) were found using a simple root-finding algorithm in a simulation restricted to the appropriate extremes of the order parameter. Both energy modes were calculated as single windows, while the solid and fluid tether modes were split into 3 and 10 windows respectively (for the largest system size studied $N=500$ ). 


\section{APPLICATION TO THE LENNARD-JONES SYSTEM}

\section{A. Model and strategy}

We have employed the Phase Switch Monte Carlo method to study the freezing properties of particles whose interactions are described by the Lennard-Jones potential

$$
\Phi(r)=4 \epsilon\left[\left(\frac{\sigma}{r}\right)^{12}-\left(\frac{\sigma}{r}\right)^{6}\right] .
$$

The system is assumed to be contained within a cubic box of volume $V$, which fluctuates under the control of an applied external pressure $p$ and is in thermal equilibrium at temperature $T$. In common with previous studies of freezing in the LJ system [11, 23], particle interactions were truncated at one-half the box length, and running tail corrections applied to the energy in the standard manner [3]. As described in sec. III B 1, the MC moves employed were particle translations, volume moves, association swaps and phase switches. Of these, we note that volume moves and phase switches are computationally inexpensive to implement because the associated energy change is obtainable directly from the scaling with (respect to coordinate dilations) of the repulsive and attractive parts of the configurational energy [37]. Association swaps are similarly cheap because they leave the configurational energy unaltered. Since the position of one particle was clamped at its representative site in each phase, CS phase association swaps were not required (see sec. III B 1 and appendix A).

The phase switch MC method requires for its operation the specification of a representative configuration $\{\mathbf{R}\}^{\gamma}$ for both the CS and F phases. For $\{\mathbf{R}\}^{C S}$ we adopt a perfect lattice of the appropriate symmetry (here fcc) and scale (corresponding to a rough estimate of the CS phase coexistence density). For $\{\mathbf{R}\}^{F}$, we randomly select a configuration in the course of a simulation of the fluid phase [32].

With regard to the order parameter (sec sec III A), the hand-over from tether to energy mode is controlled by the parameter $\tilde{u}_{c}$ in eq. 10. This should be chosen such that -at the hand-over- particles are sufficiently close to their representative sites that the energy $\operatorname{cost} \Delta \mathcal{E}_{\gamma^{\prime} \gamma}$ of the switch (eq. 12) is not saturated at a large value. Otherwise $M_{\mathcal{E}, \gamma}$ will be unable to guide the particles to positions favorable for the phase switch. We find that satisfactory results are obtained by choosing $\tilde{u}_{c}$ consistent with $M_{\mathcal{E}, \gamma} \approx 5$ at the hand-over.

The formalism described in Secs. II and III assumes that the configurational constraint confines the CS phase to the phase space fragment in which it is initiated. While this appears to be strictly observed for F-CS coexistence in hard spheres, as studied in ref. [21], for the softer interactions of the LJ system, interchange of neighboring particles between lattice sites was observed to occur, albeit very rarely. The simplest way of dealing with this effect is to suppress it; to which end we introduced an upper bound on the particle displacements in the CS phase. We set $\left|u_{i}\right|^{C S} \leq 0.65 \sigma$, a choice which (we have verified) has a negligible effect on the equilibrium properties of the CS phase for the system sizes we have studied.

The strategy for obtaining the F-CS coexistence line is as follows. Initially one requires a rough estimate for some coexistence state point. This may derive either from any available literature estimates or, alternatively, coexistence can be bracketed by scanning (backwards and forwards) a range of pressures at some nominated temperature until the system spontaneously transforms between phases. Next it is necessary to customize a suitable set of multicanonical weights which allow the system to sample both phases. This can be done by employing, for example, the transition matrix method described in sec. III B 3. An example weight function is shown in fig. 3 for a system of $N=256$ particles close to coexistence at $\beta=\epsilon / k T=0.6$.

Having determined a suitable weight function, a production run is then performed to obtain data of high statistical quality. From this data one can readily extract (see sec. III B 2) the ratio $\mathcal{R}_{\mathrm{F}, \mathrm{CS}}$ (eq. 17) and hence the free energy difference $\Delta g$ (eq. 9) between the phases. Histogram extrapolation (HE) (sec. III B 2) then permits the location of the coexistence pressure at which this difference vanishes (i.e. when $\mathcal{R}_{\mathrm{F}, \mathrm{CS}}=1$ ). The corresponding coexistence number densities can then be simply read off from the peak positions in the coexistence form of $P(\rho)$, where $\rho=N / V$. Fig. 4 shows a typical coexistence form of $P(\rho)$, obtained for $N=256$ at $\beta=0.6$.

In addition to simplifying the determination of the coexistence pressure at a given temperature, HE also permits simultaneous extrapolation in the temperature. This facilitates the scanning of a segment of the coexistence line in the $p-T$ plane. Although the range of near coexistence $p-T$ values for which a single simulation provides reliable information is necessarily limited, additional simulations can be performed near the vestiges of this range. The data from simulations performed at a number of different state points can then be synthesised self-consistently using multiple histogram reweighting [33]. As well as guiding the choice of near coexistence values of $p$ and $T$ for subsequent simulations, HE also provides an estimate of the appropriate order parameter distribution $P\left(M_{m, \gamma}\right)$, which (recall sec. III B 3) serves as a suitable weight function. Thus by a repeated combination of simulation followed by 


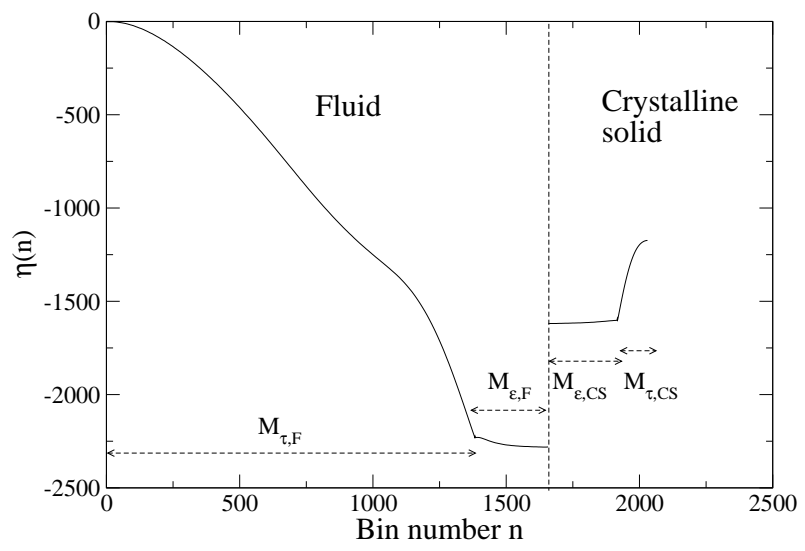

FIG. 3: An example weight function used for a near-coexistence production run. The data shown are for a system of $N=256$ LJ particles at $\beta=0.6, P=13.7$. The four branches of the weight function have been laid out with respect to the underlying discretisation into bins (see sec. III B 3).

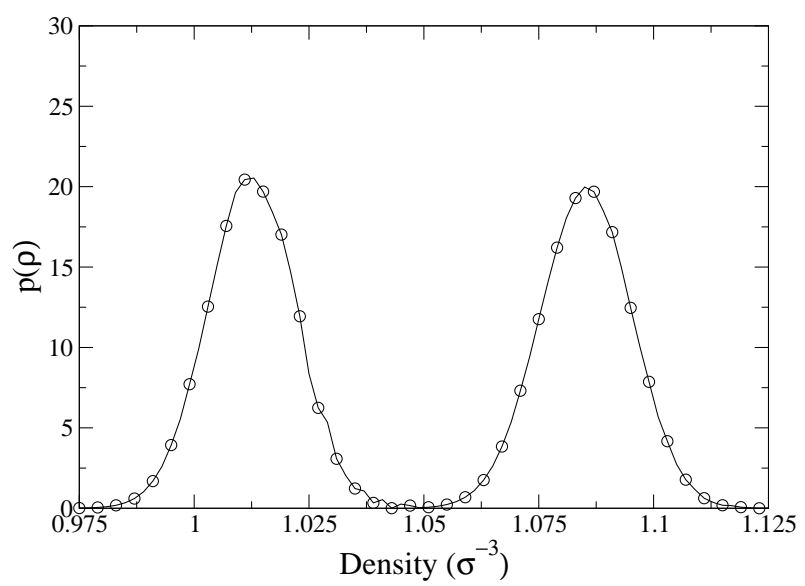

FIG. 4: The coexistence form of the density distribution $P(\rho)$ for a system of $N=256$ LJ particles at $\beta=0.6, p=13.722$. A selection of data points are shown. Lines are guides to the eye.

$\mathrm{HE}$, the coexistence line can be straightforwardly tracked, without the need to ever re-determine a weight function ab initio [31].

\section{B. Results}

Estimates of the freezing boundary have been obtained using the PSMC method within the constant- $N p T$ ensemble for systems of sizes $N=108,256$ and 500 particles. Freezing boundary data has also been obtained for $N=32$, but here the system is sufficiently small that transitions back and forth between F and CS phases occur spontaneously, over a range of pressures, and a density distribution (sampling both phases) can be determined -and a coexistence pressure inferred- without the need for PSMC.

Spontaneous transitions were also found to occur (albeit much more rarely) in the $N=108$ system. Here we observed spontaneous freezing as we tracked the coexistence curve using the methods described in sec. IV A. We emphasize that this effect was also seen for this system size in a 'bare' constant- $N p T$ simulation at coexistence and does not therefore appear to be caused (or rendered more frequent) by the multicanonical weighting of PSMC. The larger system sizes $(N=256$ and $N=500)$ did not exhibit spontaneous transitions on the timescale of our simulations, presumably because of the increased free energy cost of forming an interface.

The spontaneous freezing that occurs in the $N=108$ system undermines our strategy whereby the phase label $\gamma$ keeps track of the current phase. Nevertheless, we were able to suppress the problem by tracking the coexistence line along a path parallel to its true trajectory, but displaced slightly towards the fluid side. This was achieved by simulating at a pressure some $5 \%$ less than the coexistence value predicted by the histogram extrapolation from the 
previous near-coexistence state point. The estimate of the coexistence pressure was easily corrected for the imposed shift by means of histogram extrapolation.
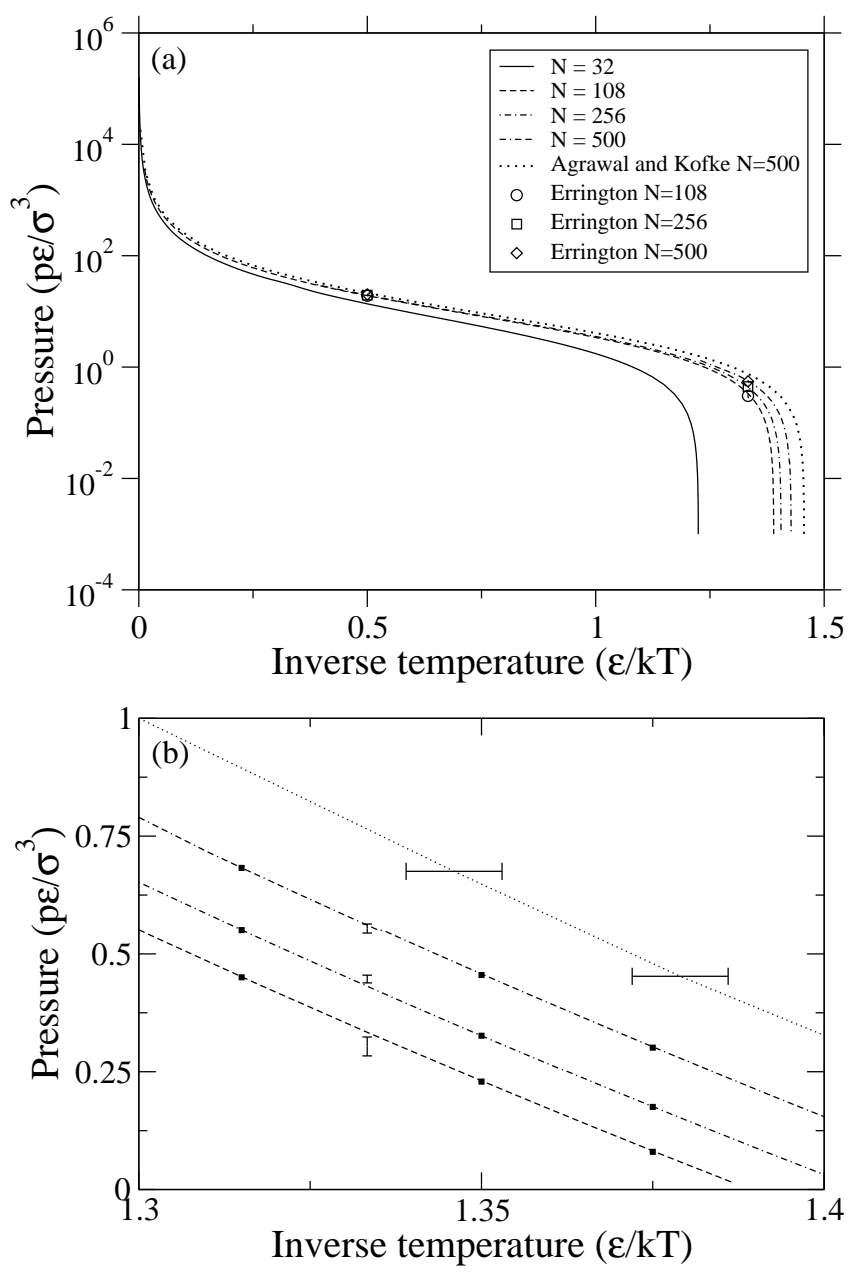

FIG. 5: (a) The estimated F-CS phase diagram in the pressure-inverse temperature plane for the four systems sizes studied in this work. The data shown derive from 20 separate simulation state points for the $N=32$ system size, 37 state points for $N=108,17$ points for $N=256$ and 4 points for the $N=500$ system size. Also included for comparison are the PSMC estimates of Errington [23] for $\beta=4 / 3,1 / 2$, together with the GDI results of Agrawal and Kofke [11] for $N=500$. (b) A closeup of the region around $\beta=4 / 3$. The vertical error bars corresponding to Errington's data points [23] and the horizontal error bars to the GDI study. Symbols are the estimates arising from the present study (given explicitly in tables I-III), with uncertainties smaller than the symbol size in each case. Lines are interpolations between the data points, based on histogram extrapolation.

The measured phase boundary in the $p-T$ plane, and a portion of the phase diagram in the $\rho-T$ plane, are shown in figs. 5 and 6 respectively for the various system sizes studied. Full data are also tabulated in tables I-III together with their uncertainties. With regard to the latter, we note that analysis of statistical errors within the PSMC framework is extremely transparent: at a given temperature, the uncertainty in the pressure is simply given by

$$
\sigma[p]=\frac{\sigma\left[\mathcal{R}_{\mathrm{F}, \mathrm{CS}}\right]}{|\Delta \bar{V}|}
$$

where $\Delta \bar{V}=\bar{V}_{F}-\bar{V}_{C S}$, and $\sigma\left[\mathcal{R}_{\mathrm{F}, \mathrm{CS}}\right]$ is the associated uncertainty in $\mathcal{R}_{\mathrm{F}, \mathrm{CS}}$, this being controlled (at heart) by the statistics of the inter-phase switch and measurable from a simple block error analysis. We note that owing to the extensive factor $\Delta \bar{V}$ in the denominator of eq. 32, the minimum uncertainty in $\mathcal{R}_{\mathrm{F}, \mathrm{CS}}$ required to attain a prescribed error in the pressure grows like $N[38]$. 


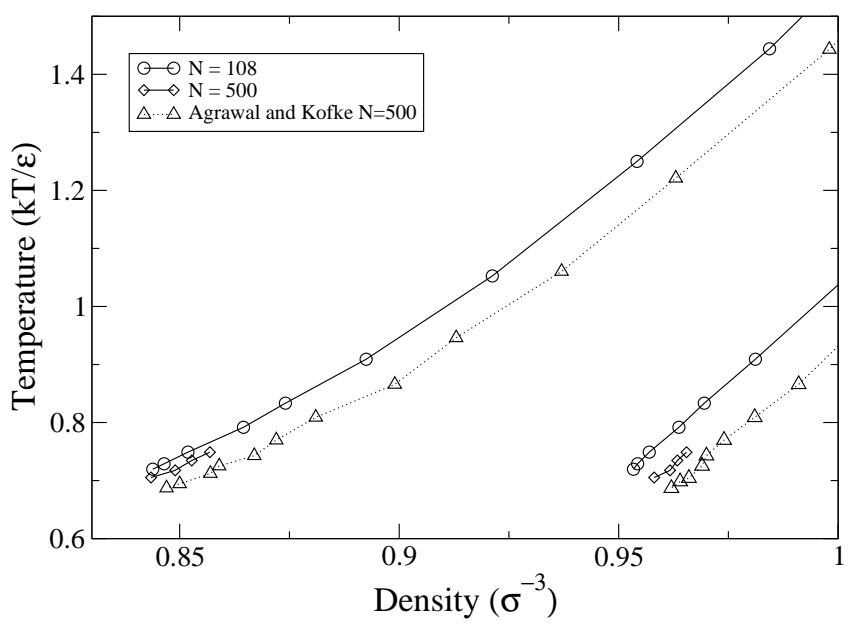

FIG. 6: A portion of the phase diagram in the $\rho-T$ plane in the region of the triple point. Shown are the estimated solid and fluid coexistence densities for $N=500$ and $N=108$. Also included for comparison are the GDI estimates of Agrawal and Kofke[11] for $N=500$. Uncertainties are smaller than the symbol sizes; lines are guides to the eye

As with any method, the error analysis becomes more involved if one chooses to combine a number of separate data sets via a multiple histogram analysis [33]. In these circumstances we have found it useful to perform a bootstrap error analysis with 100 re-samples, taking an estimate of the block size as the correlation length. The error estimate was found to depend only weakly on the block size. All error bars in tables I-III are calculated in this way and correspond to a $67 \%$ confidence interval. We stress that it is not strictly necessary to perform this more sophisticated error analysis, adequate (albeit slightly overly conservative) error bars can be assigned simply on the basis of block averages.

Ideally one should like to extrapolate coexistence parameters obtained for a range of finite system sizes to the thermodynamic limit using a finite-size scaling ansatz. In the hard sphere case, a clear $N^{-1}$ dependence of the coexistence pressure has been observed using the PSMC method [21, 23], and there seems no reason to expect a different scaling in the case of the LJ potential. However, in the present study (as well as in that of ref. [23]), a good fit to this scaling form could not be obtained. As noted in ref. [23], the reason for this is presumably traceable to the truncation of the interparticle potential cutoff, which was set to be one-half the box length in all cases. This choice, while facilitating direct comparison with existing literature data (principally ref. [11]), necessarily introduces a degree of coupling between the potential truncation and the system size, notwithstanding the use of a mean-field based tail correction. For the rather limited range of system sizes studied in this work, the use of such a tail correction is unlikely to provide a good approximation to the full potential, particularly for the smaller system sizes. Indeed, it is well known that many aspects of the phase behaviour of the LJ system are acutely sensitive to the details of the potential cutoff $[23,28,40]$.

\section{DISCUSSION AND CONCLUSIONS}

In summary, the Phase Switch MC method allows one to locate -directly and transparently- fluid-solid coexistence parameters and their associated uncertainties within the appropriate (constant-pressure) ensemble. To achieve this the method connects the phases via a direct inter-phase sampling path, thereby facilitating estimates of free energy differences from a single simulation. The course of this path encompasses configurations which are exclusively purephase in character. Accordingly, one can be confident that it robust, i.e. free of both singularities and crystalline defects.

We have applied the PSMC method to calculate, for a number of system sizes, significant portions of the $p-T$ freezing curve of the LJ fluid to high accuracy (see the error bars quoted in tables I-III). In so doing we have illustrated the strategic advantages of histogram extrapolation (HE) as an efficient means of tracking the coexistence line. Furthermore we have seen (cf. refs. $[13,31]$ ) that HE provides reliable estimates of the requisite multicanonical weight function, thereby obviating the need to redetermine the weights from scratch at each successive near-coexistence state point. While it is probably fair to say that the PSMC remains a somewhat computationally intensive strategy (at least in the context of the F-CS problem), it is by no means prohibitively so on the scale of its competitors. Given the strengths of the method we have identified, we feel it should represent an attractive option, especially when high 


\begin{tabular}{|c|c|c|c|c|c|}
\hline \multicolumn{6}{|c|}{$N=108$} \\
\hline$\epsilon / k T$ & $p \sigma^{3} / \epsilon$ & $\rho_{C S} \sigma^{3}$ & $\rho_{F} \sigma^{3}$ & $\mathcal{E}_{C S} / N \epsilon$ & $\mathcal{E}_{F} / N \epsilon$ \\
\hline 0.01250 & $3489(8)$ & $2.5682(18)$ & $2.5531(10)$ & $1563.4(6)$ & $1572.15(31)$ \\
\hline 0.01550 & $2635(9)$ & $2.4141(11)$ & $2.3277(13)$ & $1204.7(3)$ & $1251.84(54)$ \\
\hline 0.01900 & $2039(3)$ & $2.3045(4)$ & $2.2197(2)$ & $1008.5(1)$ & $1050.32(8)$ \\
\hline 0.02350 & $1549(2)$ & $2.1905(2)$ & $2.1091(1)$ & $813.48(7)$ & $847.951(32)$ \\
\hline 0.02800 & $1232(1)$ & $2.1014(3)$ & $2.0212(5)$ & $632.69(7)$ & $660.221(73)$ \\
\hline 0.03500 & $922(1)$ & $1.9935(2)$ & $1.9182(3)$ & $503.94(5)$ & $526.283(37)$ \\
\hline 0.04500 & $663.1(9)$ & $1.8803(2)$ & $1.8078(3)$ & $389.12(4)$ & $406.995(38)$ \\
\hline 0.05500 & $507.7(8)$ & $1.7935(1)$ & $1.7235(2)$ & $312.12(2)$ & $326.930(22)$ \\
\hline 0.06500 & $406.5(6)$ & $1.7260(2)$ & $1.6576(1)$ & $257.63(2)$ & $270.276(10)$ \\
\hline 0.07500 & $334.6(9)$ & $1.6702(7)$ & $1.6033(3)$ & $219.03(6)$ & $230.064(21)$ \\
\hline 0.09000 & $262.0(7)$ & $1.6026(7)$ & $1.5383(5)$ & $169.65(3)$ & $178.562(29)$ \\
\hline 0.11000 & $197.7(4)$ & $1.5299(3)$ & $1.4659(2)$ & $135.32(2)$ & $142.881(16)$ \\
\hline 0.13000 & $157.8(3)$ & $1.4773(3)$ & $1.4142(2)$ & $110.82(2)$ & $117.339(8)$ \\
\hline 0.16000 & $117.2(2)$ & $1.4111(4)$ & $1.3477(6)$ & $82.416(15)$ & $87.742(24)$ \\
\hline 0.17500 & $103.0(3)$ & $1.3827(3)$ & $1.3213(5)$ & $72.448(1)$ & $77.294(24)$ \\
\hline 0.21000 & $78.95(13)$ & $1.3304(3)$ & $1.2692(1)$ & $56.230(9)$ & $60.335(4)$ \\
\hline 0.24000 & $64.68(8)$ & $1.2940(1)$ & $1.2327(1)$ & $47.043(4)$ & $50.738(5)$ \\
\hline 0.30000 & $45.66(6)$ & $1.2349(1)$ & $1.1734(4)$ & $30.560(4)$ & $33.483(7)$ \\
\hline 0.37000 & $32.43(5)$ & $1.1835(1)$ & $1.1214(1)$ & $22.669(2)$ & $25.210(3)$ \\
\hline 0.43000 & $25.12(4)$ & $1.1505(11)$ & $1.0863(4)$ & $16.44(50)$ & $18.718(10)$ \\
\hline 0.50000 & $19.13(3)$ & $1.1180(11)$ & $1.0525(3)$ & $11.17(34)$ & $13.163(6)$ \\
\hline 0.62000 & $12.56(4)$ & $1.0762(12)$ & $1.0070(3)$ & $5.21(16)$ & $6.908(4)$ \\
\hline 0.69257 & $9.840(17)$ & $1.0557(11)$ & $0.9844(2)$ & $2.72(8)$ & $4.271(3)$ \\
\hline 0.80000 & $6.859(8)$ & $1.0291(11)$ & $0.9542(2)$ & $-0.23(1)$ & $1.157(3)$ \\
\hline 0.95000 & $4.105(10)$ & $1.0023(11)$ & $0.9212(1)$ & $-2.98(91)$ & $-1.735(2)$ \\
\hline 1.10000 & $2.236(7)$ & $0.9812(11)$ & $0.8925(2)$ & $-4.85(15)$ & $-3.701(2)$ \\
\hline 1.20000 & $1.293(4)$ & $0.9695(11)$ & $0.8741(1)$ & $-5.86(18)$ & $-4.752(1)$ \\
\hline $1.26282(42)$ & 0.8173 & $0.9637(11)$ & $0.8645(1)$ & $-6.30(19)$ & $-5.207(1)$ \\
\hline $1.33510(54)$ & 0.3150 & $0.9570(11)$ & $0.8519(2)$ & $-6.83(21)$ & $-5.755(1)$ \\
\hline $1.37193(39)$ & 0.1003 & $0.9543(11)$ & $0.8464(3)$ & $-7.07(21)$ & $-5.994(2)$ \\
\hline $1.38077(56)$ & 0.0466 & $0.9537(11)$ & $0.8447(2)$ & $-7.13(21)$ & $-6.051(2)$ \\
\hline $1.38564(46)$ & 0.0250 & $0.9537(11)$ & $0.8447(2)$ & $-7.15(22)$ & $-6.080(1)$ \\
\hline $1.38674(47)$ & 0.0150 & $0.9533(11)$ & $0.8438(2)$ & $-7.16(22)$ & $-6.087(2)$ \\
\hline $1.38963(91)$ & 0.0010 & $0.9534(11)$ & $0.8439(1)$ & $-7.18(22)$ & $-6.105(1)$ \\
\hline
\end{tabular}

TABLE I: Solid-fluid coexistence curve data for the $N=108$ system. Tabulated in columns $1-6$, respectively, are the inverse freezing temperature, pressure, solid number density, fluid number density, energy per particle (solid) and energy per particle (fluid). Numbers in parentheses indicate the the $67 \%$ confidence limit for the rightmost digit(s). Note that due to the steepness of the coexistence curve at low pressures (cf. fig. 5), it is expedient in this regime to determine the coexistence temperature at prescribed pressure.

precision estimates of freezing boundaries are required.

We have compared our results with those of the previous PSMC study of Errington [23] and the GDI study of Agrawal and Kofke [11] for $N=500$. Not surprisingly, our results agree (to within error) with those of Errington. However, (and as also noted by Errington), the results for $N=500$ do not overlap with the error bars quoted in the GDI study of the same system size (and potential truncation) by Agrawal and Kofke [11]. This is possibly due to the inherent limitations of the GDI scheme as already noted in sec. I -specifically the lack of any means to reconnect the phases beyond the initial 'starting' coexistence state point from where the integration is launched. It may additionally reflect a poor estimate of the starting point itself, which was determined using TI by transforming the LJ system to a hard sphere reference system via a route which takes in an intermediate system of soft spheres. In particular, the coexistence pressure taken for the hard sphere reference system is now believed to be an overestimate [21, 23, 41]. 


\begin{tabular}{llllll}
\hline \multicolumn{6}{c}{$N=256$} \\
\multicolumn{1}{c}{$\epsilon / k T$} & \multicolumn{1}{c}{$p \sigma^{3} / \epsilon$} & $\rho_{C S} \sigma^{3}$ & $\rho_{F} \sigma^{3}$ & $\mathcal{E}_{C S} / N \epsilon$ & \multicolumn{1}{c}{$\mathcal{E}_{F} / N \epsilon$} \\
\hline 0.39000 & $30.400(47)$ & $1.1771(3)$ & $1.10965(3)$ & $20.200(22)$ & $22.8182(6)$ \\
0.43000 & $25.390(180)$ & $1.1525(5)$ & $1.08401(12)$ & $15.870(22)$ & $18.2534(30)$ \\
0.47000 & $21.942(37)$ & $1.1355(2)$ & $1.06611(1)$ & $12.430(13)$ & $14.6269(1)$ \\
0.54000 & $16.928(34)$ & $1.1065(2)$ & $1.0355(2)$ & $8.1409(89)$ & $10.0970(21)$ \\
0.60000 & $13.667(49)$ & $1.0854(3)$ & $1.0130(2)$ & $5.4899(69)$ & $7.2971(19)$ \\
0.68000 & $10.470(23)$ & $1.0616(2)$ & $0.9861(5)$ & $3.1561(39)$ & $4.8445(52)$ \\
0.68000 & $10.477(18)$ & $1.0619(4)$ & $0.9864(1)$ & $3.1494(43)$ & $4.8415(15)$ \\
0.80000 & $7.068(12)$ & $1.0332(3)$ & $0.9532(1)$ & $-0.0465(6)$ & $1.4633(7)$ \\
0.91000 & $4.885(13)$ & $1.0126(3)$ & $0.9278(1)$ & $-2.1525(28)$ & $-0.7639(17)$ \\
1.00000 & $3.546(9)$ & $0.9990(3)$ & $0.9098(1)$ & $-3.5496(44)$ & $-2.2385(8)$ \\
1.10000 & $2.340(9)$ & $0.9851(3)$ & $0.8901(1)$ & $-4.7187(56)$ & $-3.4660(11)$ \\
1.20000 & $1.418(6)$ & $0.9745(3)$ & $0.8734(1)$ & $-5.7189(62)$ & $-4.5181(11)$ \\
$1.29969(94)$ & 0.6456 & $0.9651(3)$ & $0.8560(3)$ & $-6.5252(70)$ & $-5.3518(26)$ \\
$1.33481(74)$ & 0.4263 & $0.9628(3)$ & $0.8508(1)$ & $-6.7631(74)$ & $-5.5961(9)$ \\
$1.36274(71)$ & 0.2500 & $0.9604(3)$ & $0.8463(2)$ & $-6.9512(74)$ & $-5.7908(18)$ \\
$1.38853(64)$ & 0.1000 & $0.9587(3)$ & $0.8422(2)$ & $-7.1148(76)$ & $-5.9582(11)$ \\
$1.40025(60)$ & 0.0300 & $0.9578(3)$ & $0.8405(1)$ & $-7.1896(77)$ & $-6.0372(8)$ \\
$1.40527(68)$ & 0.0010 & $0.9574(3)$ & $0.8395(1)$ & $-7.2208(77)$ & $-6.0681(6)$ \\
\hline
\end{tabular}

TABLE II: Solid-fluid coexistence curve data for $N=256$. See caption of tab. I for details.

\begin{tabular}{cccccc}
\hline \multicolumn{6}{c}{$N=500$} \\
$\epsilon / k T$ & $p \sigma^{3} / \epsilon$ & $\rho_{C S} \sigma^{3}$ & $\rho_{F} \sigma^{3}$ & $\mathcal{E}_{C S} / N \epsilon$ & $\mathcal{E}_{F} / N \epsilon$ \\
\hline $1.336(2)$ & 0.5500 & $0.9655(1)$ & $0.8566(2)$ & $-6.637(1)$ & $-5.486(2)$ \\
$1.3613(3)$ & 0.3836 & $0.96337(4)$ & $0.85274(3)$ & $-6.815(1)$ & $-5.6705(2)$ \\
$1.393(1)$ & 0.2091 & $0.9617(1)$ & $0.8490(1)$ & $-7.007(1)$ & $-5.871(1)$ \\
$1.418(2)$ & 0.0200 & $0.9581(1)$ & $0.843(1)$ & $-7.198(1)$ & $-6.08(1)$ \\
\hline
\end{tabular}

TABLE III: Solid-fluid coexistence curve data for $N=500$. See caption of tab. I for details.

As regards the prospects for further applications, it would certainly be worthwhile in the specific case of the LJ system to attempt to decouple the system size scaling of the cutoff from intrinsic finite-size effects by repeating the present study for a constant interparticle potential cutoff. This should permit a reliable extrapolation of coexistence parameters to the thermodynamic limit, albeit for the particular choice of cutoff. More generally, it would be interesting to attempt to apply the PSMC method to molecular systems. Here, however, the order parameter would probably need to be augmented in order to not only draw molecules to their representative sites, but also to align them appropriately.

\section{Acknowledgement}

The authors thank Jeff Errington for providing numerical data from ref. [23].

\section{APPENDIX A: ACCEPTANCE CRITERIA}

Here we derive the Monte Carlo acceptance probabilities used in the PSMC method.

As described in the sec. III, we choose to fix one particle at its representative site. The effect of this is to reduce the configurational weight of each phase by a factor of the system volume $V$. Thus for the fluid phase, eq. 4 becomes 


$$
Z_{F}(N, V, T)=V \prod_{i=1}^{N-1} \int_{V, F} d \mathbf{u}_{i} e^{-\Phi\left(\{\mathbf{u}\}_{\star},\{\mathbf{R}\}^{F}\right)},
$$

while for the CS phase (using eq. 5), we have

$$
Z_{C S}(N, V, T)=(N-1) ! V \prod_{i=1}^{N-1} \int_{V, C S} d \mathbf{u}_{i} e^{-\Phi\left(\{\mathbf{u}\}_{*},\{\mathbf{R}\}^{C S}\right)} .
$$

In analogy to eq. 13 the phase space relevant to the problem is described by the multicanonical ensemble

$$
\tilde{\mathcal{Z}}(N, p, T,\{\eta\}) \equiv \sum_{\gamma} \int_{0}^{\infty} V d V \prod_{i=1}^{N-1} \int_{\gamma} d \mathbf{u}_{i} e^{-\mathcal{H}_{\gamma}\left(\{\mathbf{u}\}_{\star}, V\right)}
$$

where

$$
\begin{aligned}
\mathcal{H}_{\gamma}\left(\{\mathbf{u}\}_{\star}, V\right)= & \Phi\left(\{\mathbf{u}\}_{\star},\{\mathbf{R}\}^{\gamma}\right)+p V+ \\
& \eta_{m, \gamma}(M)-\delta_{\gamma, C S} \ln (N-1) !
\end{aligned}
$$

The configurational probability follows as

$$
P\left(\{\mathbf{u}\}_{\star}, V, \gamma \mid N, p, T,\{\eta\}\right)=\frac{V e^{-\mathcal{H}_{\gamma}\left(\{\mathbf{u}\}_{\star}, V\right)}}{\tilde{\mathcal{Z}}(N, p, T,\{\eta\})}
$$

Consider now a MC move (particle translation, association swaps, or volume move) which leaves the phase label unchanged. Let

$$
P_{A} \equiv P\left(\{\mathbf{u}\}_{\star}, V\right) \prod_{i=1}^{N-1} d \mathbf{u}_{i} d V
$$

be the probability that the system is found in the region of configuration space of volume $\prod_{i} d \mathbf{u}_{i} d V$ around $\{\mathbf{u}\}_{\star}, V$. Similarly let

$$
P_{B} \equiv P\left(\{\mathbf{u}\}_{\star}^{\prime}, V^{\prime}\right) \prod_{i=1}^{N-1} d \mathbf{u}_{i}^{\prime} d V^{\prime}
$$

represent the probability associated with the range of configuration space reached by implementing a change $\left(\{\mathbf{u}\}_{\star}, V\right) \rightarrow\left(\{\mathbf{u}\}_{\star}^{\prime}, V^{\prime}\right)$. Detailed balance requires

$$
\frac{p_{a}(A \rightarrow B)}{p_{a}(B \rightarrow A)}=\frac{P_{B}}{P_{A}}
$$

where

$$
\frac{P_{B}}{P_{A}}=\frac{V^{\prime} \exp \left[-\mathcal{H}_{\gamma}\left(\{\mathbf{u}\}_{\star}^{\prime}, V^{\prime}\right)\right] \prod_{i=1}^{N-1} d \mathbf{u}_{i}^{\prime} d V^{\prime}}{V \exp \left[-\mathcal{H}_{\gamma}\left(\{\mathbf{u}\}_{\star}, V\right)\right] \prod_{i=1}^{N-1} d \mathbf{u}_{i} d V} .
$$

Now for a volume update that increments the current value, $d V=d V^{\prime}$, while

$$
\frac{V^{\prime} \prod_{i=1}^{N-1} d \mathbf{u}_{i}^{\prime}}{V \prod_{i=1}^{N-1} d \mathbf{u}_{i}}=\left[\frac{V^{\prime}}{V}\right]^{N}
$$


Thus

$$
\frac{p_{a}(A \rightarrow B)}{p_{a}(B \rightarrow A)}=\frac{\exp \left[-\mathcal{H}_{\gamma}\left(\{\mathbf{u}\}_{\star}^{\prime}, V^{\prime}\right)+N \ln V^{\prime}\right]}{\exp \left[-\mathcal{H}_{\gamma}\left(\{\mathbf{u}\}_{\star}, V\right)+N \ln V\right]}
$$

from which the acceptance probability follows as

$$
\begin{aligned}
& p_{a}\left(\{\mathbf{u}\}_{\star}, V \rightarrow\{\mathbf{u}\}_{\star}^{\prime}, V^{\prime} \mid \gamma\right)= \\
& \quad \min \left\{1, \exp \left[-\Delta \mathcal{H}_{\gamma}+N \ln \left(V^{\prime} / V\right)\right]\right\}
\end{aligned}
$$

with

$$
\Delta \mathcal{H}_{\gamma}=\mathcal{H}_{\gamma}\left(\{\mathbf{u}\}_{\star}^{\prime}, V^{\prime}\right)-\mathcal{H}_{\gamma}\left(\{\mathbf{u}\}_{\star}, V\right) .
$$

We note that the acceptance formula eq. A11 is actually no different to that pertaining to a fully unconstrained constant- $N p T$ ensemble [3]. Indeed a little thought reveals that the act of fixing one particle is simply equivalent to viewing the unconstrained system from the reference frame of that particle. Such a change of reference frame has no consequences for acceptance criteria.

Consider next the inter-phase switch. This replaces one set of representative vectors, $\{\mathbf{R}\}^{\gamma}$ say, by the other, $\{\mathbf{R}\}^{\gamma^{\prime}}$, while at the same time scaling the system volume and particle displacements by a constant factor chosen such that a 'typical' volume $\hat{V}_{\gamma}$ of phase $\gamma$ is matched to a 'typical' volume $\hat{V}_{\gamma^{\prime}}$ of phase $\gamma^{\prime}$. For a volume update which scales the current value, one has $d V^{\prime} / d V=V^{\prime} / V$, and referring back to the detailed balance relation eq. A9 one finds

$$
\frac{V^{\prime} \prod_{i=1}^{N-1} d \mathbf{u}_{i}^{\prime} d V^{\prime}}{V \prod_{i=1}^{N-1} d \mathbf{u}_{i} d V}=\left[\frac{V^{\prime}}{V}\right]^{N+1} .
$$

The switch acceptance probability follows as

$$
\begin{aligned}
& p_{a}\left(\gamma,\{\mathbf{u}\}_{\star}, V \rightarrow \gamma^{\prime},\{\mathbf{u}\}_{\star}^{\prime}, V^{\prime}\right)= \\
& \quad \min \left\{1, \exp \left[\Delta \mathcal{H}_{\gamma^{\prime} \gamma}+(N+1) \ln \left(\hat{V}_{\gamma^{\prime}} / \hat{V}_{\gamma}\right)\right]\right\}
\end{aligned}
$$

where

$$
\Delta \mathcal{H}_{\gamma \gamma^{\prime}}=\mathcal{H}_{\gamma^{\prime}}\left(\{\mathbf{u}\}_{\star}^{\prime}, V^{\prime}\right)-\mathcal{H}_{\gamma}\left(\{\mathbf{u}\}_{\star}, V\right) .
$$

Note that a different formulation of the switch acceptance probability applies when the displacement vectors are held constant during the switch operation $[21,23,30]$.

[1] P.A. Monson and D.A. Kofke, Adv. Chem. Phys. 115, 113 (2000).

[2] D. Frenkel and A.J.C. Ladd, J. Chem. Phys. 81, 3188 (1984).

[3] D. Frenkel and B. Smit, Understanding Molecular Simulation, 2nd edition. (Academic, San Diego, 2002).

[4] This is evidenced by the persistence of attempts to develop 'other' approaches.

[5] A.D. Bruce and N.B. Wilding, Adv. Chem. Phys. 127, 1 (2003).

[6] Eg the singular behaviour in the single-occupancy-cell (SOC) model: H. Ogura, M Matsuda, T. Ogawa, N. Ogita and A. Ueda, Prog. Theor. Phys., 58, 419 (1977). For anything but hard spheres, in traversing the fluid phase there is also a liquid-vapor binodal to be circumnavigated.

[7] J.M. Polson , E. Trizac, S. Pronk and D. Frenkel, J. Chem. Phys., 112 , 5339 (2000)

[8] For a recent application of TI to the F-CS problem, see M.A. Barroso and A.L. Ferreira, J. Chem. Phys. 116, 7145 (2002).

[9] D.A. Kofke, Mol. Phys. 78, 1331 (1993).

[10] D.A. Kofke, J. Chem. Phys. 98, 4149 (1993).

[11] R. Agrawal and D.A. Kofke, Mol. Phys. 85, 43 (1995).

[12] R. Agrawal and D.A. Kofke, Phys. Rev. Lett. 74, 122 (1995); D.A. Kofke and P.G. Bolhuis, Phys. Rev. E 59 618 (1999); F.A. Escobedo and J.J. de Pablo J. Chem. Phys. 1062911 (1997).

[13] N.B. Wilding, Phys. Rev. E 52, 602 (1995). 
[14] G. Grochla, J. Chem. Phys. 120, 2122 (2004).

[15] D.M. Elke, J.F. Brennecke and E. J. Maginn, J. Chem. Phys. 122, 014115 (2005).

[16] G. Grochla, J. Chem. Phys. 122, 046101 (2005).

[17] E.A. Mastny and J.J. de Pablo, J. Chem. Phys. 122, 124109 (2005).

[18] The actual implementation swaps replicas between the overlapping windows. These swaps are accepted with probability unity whenever two adjacent replicas are in the overlap region, and are rejected otherwise. In the central region, which has two exactly overlapping windows - one for liquid and one for solid- trail swaps are accepted with unit probability, yielding a 'density of states' which is the simple average of those for the solid and liquid branches.

[19] At the time of writing, the authors of ref. [17] are investigating whether repeated transitions between the two phases occur in their method: J.J. de Pablo, personal communication.

[20] F.A. Escobedo, J. Chem. Phys. 123, 044110 (2005).

[21] N.B. Wilding and A.D. Bruce, Phys. Rev. Lett. 85, 5138 (2000).

[22] A.D. Bruce, N.B. Wilding and G.J. Ackland, Phys. Rev. Lett., 79, 3002 (1997); A.D. Bruce, A.N. Jackson, G.J. Ackland and N.B. Wilding, Phys. Rev. E, 61, 906 (2000).

[23] J.R. Errington, J. Chem. Phys. 120, 3130 (2004).

[24] $p, V$ and $\Phi$ are expressed in units of $k T / \sigma^{3}, \sigma^{3}$ and $k T$ respectively, where for a given interparticle potential $\sigma$ sets the particle length scale, cf. eq. 31.

[25] At our starting point (Eq. 2) the indices $1 \ldots N$ on the coordinates $\{\mathbf{r}\}$ are particle labels which confer distinguishability. Any 'correction for indistinguishability' is made post hoc. With this perspective the 'fragments' are 'distinct'.

[26] Essentially, the global translation mode can be regarded as allowing the system to access $N$ permutations of the particles with respect to the underlying notional fixed lattice. Doing so reduces the actual number of permutations from $N$ ! to $(N-1)$ !. This point (first emphasized in ref. [21]) appears to have been neglected in previous studies of CS phase free energies.

[27] Unless the fluid phase configuration is 'close' to the representative one, the switch may create defective or glassy (not crystalline) configurations.

[28] A.N. Jackson, A.D. Bruce, and G.J. Ackland, Phys. Rev. E 65, 036710 (2002).

[29] B.A. Berg and T. Neuhaus, Phys. Rev. Lett., 68, 9 (1992); for an overview see [5].

[30] In this work, the scaling at the switch is applied to the box size, the representative configuration vectors and the displacement vectors. Previous work [21, 23] chose to keep the displacement vectors constant at the switch. Both methods are legitimate provided that the consequences for the switch acceptance probability (see appendix. A) are accommodated.

[31] N.B. Wilding, Am. J. Phys. 69, 1147 (2001).

[32] To ensure that no two sites of $\{\mathbf{R}\}^{F}$ are atypically close to one another (which might otherwise impede access to the gateway states), we 'anneal' this configuration before adopting it. This entails quenching the system (at constant volume), while suppressing crystallization by constraining particles to remain within a maximum displacement $0.3 \sigma$ from their starting point.

[33] A.M. Ferrenberg and R.H. Swendsen, Phys. Rev. Lett., 61, 2635 (1988); ibid 63, 1195 (1989).

[34] G.R. Smith and A.D. Bruce, J. Phys. A28, 6623 (1995).

[35] M. Fitzgerald, R.R. Picard, R.N. Silver, Europhys. Lett. 46, 282 (1999).

[36] M. Fitzgerald, R.R. Picard, R.N. Silver, J. Stat. Phys. 98, 321 (2000).

[37] M.P. Allen and D.J. Tildesley, Computer Simulation of Liquids, Oxford (1988).

[38] Coexistence parameters are determined inherently more accurately by the PSMC method than the number and energy densities of the respective phases (cf. eq. 32). One could therefore reduce (at relatively little cost) the uncertainties associated with the densities simply by performing additional single phase simulations at the stated coexistence parameters.

[39] W.G. Hoover and F.H. Ree, J. Chem. Phys, 49, 3609 (1968).

[40] B. Smit, J. Chem. Phys, 96, 8639 (1992).

[41] M.B. Sweatman, Phys. Rev. E72, 016711 (2005). 